\title{
Solvent-free liquid-phase synthesis of polyhydroquinoline derivatives under microwave irradiation
}

\author{
Xiao-Lan Zhang ${ }^{\text {a, b }}$, Shou-Ri Sheng ${ }^{\text {a,* }}$, Xian-Liang Liu, ${ }^{\text {a }}$ and Xiao-Ling Liu ${ }^{\text {a }}$ \\ ${ }^{a}$ College of Chemistry and Chemical Engineering, Jiangxi Normal University, Nanchang, \\ 330027, P. R. China \\ ${ }^{b}$ Department of Chemistry, Shangrao Teacher's College, Shangrao 334000, P. R. China \\ E-mail:shengsr@jxnu.edu.cn
}

\begin{abstract}
Polyhydroquinoline derivatives have been efficiently prepared on polyethylene glycol (PEG) via one-pot condensation of PEG-bound acetoacetate, 1,3-cyclohexanedione, ammonium acetate and the appropriate aromatic aldehyde in the presence of catalytic amounts of polyphosphoric acid by solvent-free microwave irradiation. The procedure provided the target compounds in excellent yield and purity with a facile work-up procedure.
\end{abstract}

Keywords: Liquid-phase organic synthesis, PEG-bound acetoacetate, polyhydroquinoline, microwave irradiation, solvent-free

\section{Introduction}

4-Substituted 1,4-dihydropyridines (1,4-DHPs) are analogues of NADH coenzymes and an important class of drugs. ${ }^{1}$ Current literature reveals that these compounds possess a variety of biological activities. ${ }^{2}$ Furthermore, hydrogenation methods for the conversion of these compounds to pyridines have been investigated intensively. ${ }^{3}$ Classical methods for the synthesis of 1,4-dihydropyridines are one-pot condensation of an aldehyde with ethyl acetoacetate and ammonium acetate, either in acetic acid or under reflux in alcohols. ${ }^{4}$ However, these methods suffer from several drawbacks such as a long reaction times, an excess of organic solvent, lower product yields, and harsh refluxing conditions. Therefore, it is necessary to develop more efficient and versatile methods for the preparation of 1,4-DHPs and the progress in this area is remarkable, including the recent use of microwaves, ${ }^{5}$ ionic liquids, ${ }^{6}$ refluxing at high temperature, ${ }^{7}$ TMSCl-NaI, ${ }^{8} \mathrm{Yb}(\mathrm{OTf})_{3},{ }^{9}$ Ceric ammonium nitrate, ${ }^{10} \mathrm{I}_{2}{ }^{11}$ and solid-phase organic synthesis (SPOS) techniques. ${ }^{12}$ Although some reactions are satisfactory in terms of yield, the use of high temperatures, expensive metal precursors, catalysts that are harmful to the environment, and long reaction times limits the use of these methods. Recently, organic synthesis 
of small molecular compounds on soluble polymers, i.e. liquid-phase organic synthesis (LPOS), has become an increasingly attractive field. ${ }^{13}$ This approach couples the advantages of homogeneous solution chemistry (high reactivity, lack of diffusion phenomena and ease of analysis without the cleavage-and-check procedures) with those of SPOS (use of excess of reagents and easy isolation and purification of the products). Moreover, owing to the homogeneity of liquid-phase reactions, the reaction conditions can be utilized readily according to the corresponding solution-phase systems without large changes, and the excess of reagents is less than that in solid-phase reactions. Among the various soluble polymers, polyethylene glycol (PEG) is the most useful and promising. As part of an ongoing research program focused on the use of PEG-supported $\beta$-keto ester in liquid-phase organic synthesis, ${ }^{14}$ we developed herein the Hantzsch reaction on PEG that offers the desired products, i.e. polyhydroquinoline derivatives, in excellent yield and high purity after the direct cleavage from PEG, maintaining the simplicity of the one-pot procedure (Scheme 1). To our knowledge, there have been no reports regarding the liquid-phase synthesis of polyhydroquinoline derivatives.

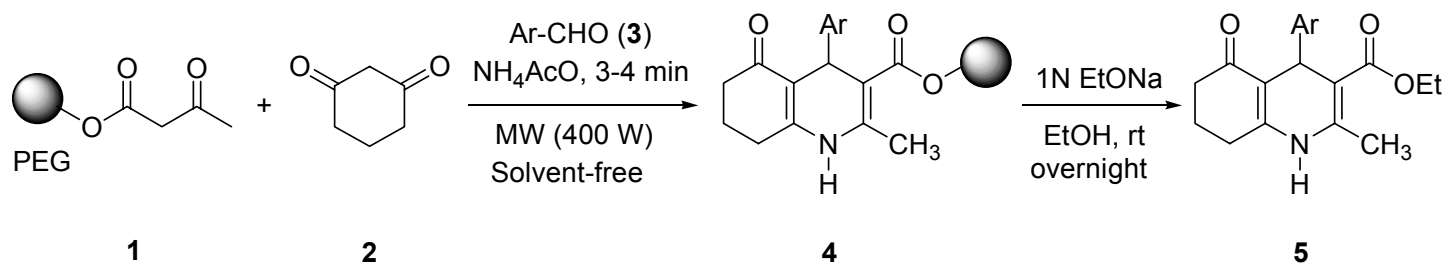

\section{Scheme 1}

\section{Results and Discussion}

The PEG 4000 bound acetoacetate 1 was prepared easily by reacting dihydroxyl PEG 4000 with 2,2,6-trimethyl-4H-1,3-dioxin-4-one in anhydrous toluene under reflux for $5 \mathrm{~h}^{14}$ The conversion of the terminal hydroxyl groups on PEG to 1 was indicated by $\mathrm{C}=\mathrm{O}$ absorptions at 1743 and $1718 \mathrm{~cm}^{-1}$ in the IR spectrum, corresponding to ester and ketone carbonyl groups, respectively, and was determined by ${ }^{1} \mathrm{H}$ NMR to be quantitative. In this liquid-phase synthetic process, the condensation of PEG-bound acetoacetate 1 with 1,3-cyclohexanedione (2), aromatic aldehydes and ammonium acetate would be the key step for the success of this protocol. First, the mixture of PEG-bound acetoacetate 1, benzaldehyde (3a), 2 and ammonium acetate was chosen as the model reaction to investigate the liquid-phase synthesis of ethyl 2-methyl-5-oxo-4-phenyl1,4,5,6,7,8- hexahydroquinoline-3-carboxylate (5a). Apparently, Lewis acids such as $\mathrm{Yb}(\mathrm{OTf})_{3},{ }^{9}$ Ceric ammonium nitrate, ${ }^{10}$ etc., could not be transferred to the PEG supported reaction system, since the Lewis acids would become ineffective due to the strong coordination between Lewis acids and the oxygen atoms on the PEG chain. Accordingly, the above mixture was stirred in 
refluxing $\mathrm{EtOH}$ or $\mathrm{AcOH}$ for $10 \mathrm{~h}$, or longer, respectively, using the classical solution-phase method. However, the condensation on PEG was not complete as monitored by FT-IR microscopy study of the PEG-bound intermediate 4a showing a new moderately strong absorption near $1690 \mathrm{~cm}^{-1}$ corresponding to the carbonyl stretch of the carbonyl group of the polyhydroquinoline ring but still exhibiting a relatively intense band near $1718 \mathrm{~cm}^{-1}$, which corresponds to the ketone carbonyl group of PEG-bound acetoacetate 1. With addition of iodine $^{11}$ into the system, the speed of the reaction was obviously accelerated, but the yield of target compound 5a cleaved from PEG was not yet satisfactory. Inspired by the results of the application of microwave (MW) irradiation, ${ }^{5}$ a better result was obtained by employing MW heating under similar reaction conditions. Interestingly, after a series of experiments, the best result was obtained in the above condensation reaction when a catalytic amount of the nonvolatile, high boiling and non-oxidizing polyphosphoric acid (PPA) was added under $400 \mathrm{~W}$ irradiation power for $3 \mathrm{~min}$ without solvent. This reaction process promoted by microwave was also monitored by FT-IR microscopy, which showed complete disappearance of the ketone carbonyl stretch near $1718 \mathrm{~cm}^{-1}$ and the appearance of a new carbonyl stretch group for the polyhydroquinoline ring near $1690 \mathrm{~cm}^{-1}$ of the PEG-bound intermediate 4a. Furthermore, this was confirmed by proton NMR spectroscopy of $4 \mathbf{a}$ since the methyl proton $\left(\mathrm{CH}_{3}\right)$ of the PEGbound acetoacetate 1 was shifted downfield from $\delta=2.27$ to $2.40 \mathrm{ppm}$. When the irradiation was stopped, the solids were treated with a small quantity of $\mathrm{CH}_{2} \mathrm{Cl}_{2}$, the PEG-bound intermediate $4 \mathbf{a}$ was then filtered and precipitated by addition of diethyl ether and was separated by filtration. The target compound 5a was obtained in 94\% yield and $97 \%$ HPLC purity of crude product by cleavage from the PEG support under the treatment of the PEG-bound product 5a with NaOEt in $\mathrm{EtOH}$ with stirring overnight. Following the synthesis of 5a on PEG, a set of related compounds (5b-5i) was prepared using an analogous synthetic protocol. As shown in Table 1, the yields (90$95 \%$ ) are excellent and the purities are satisfactory ( $\geq 95 \%$ ). It appears that there is no apparent electronic effect on the liquid-phase synthetic reaction and aromatic aldehydes with electronwithdrawing or electron-donating groups are both effective substrates. In some cases, a trace amount of the PEG residue might contaminate the final products $\mathbf{5}$, which can be easily removed by recrystallization from hot ethanol or by passing the crude product through a pad of silica gel column (10-15\% ethyl acetate in hexane as the eluent).

Additionally, cleavage of the resin-bound intermediate 4 via an alternative process was investigated as shown in Scheme 2. For example, treatment of $\mathbf{4 a}$ with $50 \% \mathrm{TFA}$ in $\mathrm{CH}_{2} \mathrm{Cl}_{2}$ at room temperature for $1 \mathrm{~h}$ furnished 2-methyl-5-oxo-4-phenyl-1,4,5,6,7,8-hexahydroquinoline-3carboxylic acid $6 a^{15}$ in $93 \%$ yield and $95 \%$ HPLC purity.

In summary, an efficient PEG supported Hantzsch reaction has been described for the liquidphase synthesis of polyhydroquinoline derivatives under solvent-free microwave promotion via one-pot four-component coupling reactions of PEG-bound acetoacetate, 1,3-cyclohexanedione, aromatic aldehydes and ammonium acetate in the presence of catalytic amounts of PPA. The process presented here is operationally simple, environmentally benign and affords excellent yields and purity, compared with the classical solution-phase reactions. 
Table 1. Yields and purities of polyhydroquinoline derivatives 5a-5i

\begin{tabular}{lllll}
\hline Entry & $\mathrm{Ar}($ Aromatic aldehydes 3) & Product & Yield (\%) $^{a}$ & Purity (\%) $^{b}$ \\
\hline 1 & $\mathrm{C}_{6} \mathrm{H}_{5}(3 \mathrm{a})$ & $5 \mathrm{a}$ & 94 & 97 \\
2 & $2-\mathrm{NO}_{2} \mathrm{C}_{6} \mathrm{H}_{4}(3 \mathrm{~b})$ & $5 \mathrm{~b}$ & 94 & 96 \\
3 & $4-\mathrm{NO}_{2} \mathrm{C}_{6} \mathrm{H}_{4}(3 \mathrm{c})$ & $5 \mathrm{c}$ & 95 & 95 \\
4 & $4-\mathrm{MeC}_{6} \mathrm{H}_{4}(3 \mathrm{~d})$ & $5 \mathrm{~d}$ & 93 & 96 \\
5 & $4-\mathrm{MeOC}_{6} \mathrm{H}_{4}(3 \mathrm{e})$ & $5 \mathrm{e}$ & 92 & 95 \\
6 & $4-\mathrm{ClC}_{6} \mathrm{H}_{4}(3 \mathrm{f})$ & $5 \mathrm{f}$ & 92 & 96 \\
7 & $4-\mathrm{FC}_{6} \mathrm{H}_{4}(3 \mathrm{~g})$ & $5 \mathrm{~g}$ & 93 & 96 \\
8 & $4-\mathrm{HOC}_{6} \mathrm{H}_{4}(3 \mathrm{~h})$ & $5 \mathrm{~h}$ & 91 & 95 \\
9 & 2-Furyl (3i) & $5 \mathrm{i}$ & 90 & 95 \\
\hline
\end{tabular}

${ }^{a}$ Isolated yield based on loading of original HO-PEG-OH; ${ }^{b}$ Determined on HPLC analysis of crude products before purification.

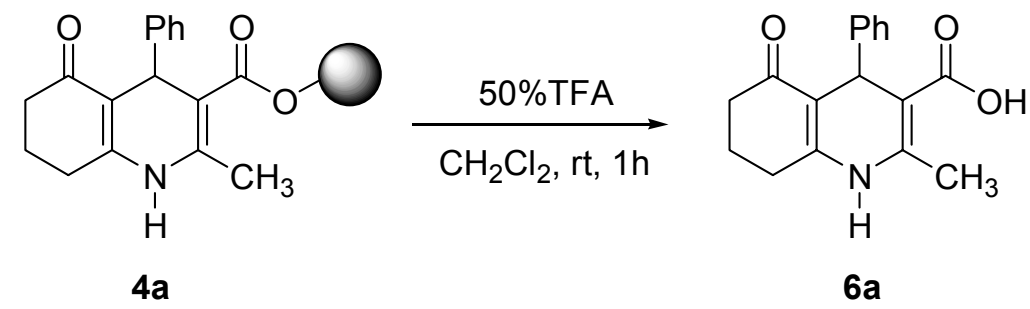

\section{Scheme 2}

\section{Experimental Section}

General Procedures. Melting points were determined on an $\mathrm{X}_{4}$ melting point apparatus and are uncorrected. ${ }^{1} \mathrm{H}$ NMR spectra were recorded on a Bruker Avance $(400 \mathrm{MHz})$ spectrometer. FTIR spectra were taken on a Perkin-Elmer SP One FT-IR spectrophotometer. Mass spectra (EI, $70 \mathrm{eV}$ ) were recorded on a HP5989B mass spectrometer. Microanalyses were performed with a PE 2400 elemental analyzer. HPLC analysis was carried out on Agilent 1100 automated system having a PDA detector $\left(\lambda_{\max }=254 \mathrm{~nm}\right)$ using a gradient from $100 \%$ of the aqueous $0.1 \%$ TFA (eluent A) to $60 \%$ eluent A- $40 \%$ of $0.5 \%$ TFA in acetonitrile (eluent B) over 35 min $(0.8$ $\mathrm{mL} / \mathrm{min})$ on a RP-18e column $(100 \times 4.6 \mathrm{~mm})$. MW experiments were performed on a Galaz WP 800J-823 domestic microwave oven. 1,3-cyclohexanedione, ammonium acetate, aromatic aldehydes were obtained from commercial sources and used without further purification.

\section{General procedure for the preparation of polyhydroquinoline derivatives (5a-5i)}

At room temperature, PPA (2-3 drops) was added to the completely ground powder of PEG 4000 bound acetoacetate acet $\mathbf{1}^{14}(1.0 \mathrm{~g}, 0.5 \mathrm{mmol}), 1,3$-cyclohexanedione $2(1.0 \mathrm{mmol})$, ammonium 
acetate $(1.2 \mathrm{mmol})$ and the corresponding aromatic aldehyde $3(1.0 \mathrm{mmol})$. The resulting mixture was added into an open vessel, stirred with a spatula for $30 \mathrm{~s}$, and the vessel was placed inside a large container filled with alumina at the center of a domestic microwave oven. After irradiation at $400 \mathrm{~W}$ for 3-4 min, the mixture was cooled to room temperature and a small volume of dichloromethane $(5 \mathrm{~mL})$ was added. After filtration, diethyl ether $(30 \mathrm{~mL})$ was added with stirring to precipitate the polymer 4 . For completion of the precipitation, the suspension was left at $0^{\circ} \mathrm{C}$ for another $30 \mathrm{~min}$. The polymer 4 was collected and washed several times with diethyl ether $(3 \times 10 \mathrm{~mL})$. After drying in vacuo, the solid was added to a $\mathrm{NaOEt}(1 \mathrm{~N}) / \mathrm{EtOH}$ solution $(15$ $\mathrm{mL}$ ) to cleave the products at room temperature overnight (checked by TLC). The target compounds 5 were obtained by extraction from the reaction mixture with EtOAc, dilution with $\mathrm{H}_{2} \mathrm{O}(20 \mathrm{~mL})$ and then removal of the solvent. The crude yellow products were purified further by recrystallization from hot ethanol or by passing the crude product through a pad of silica gel (10-15\% ethyl acetate in hexane) to give a yellow or brown solid, if necessary.

Ethyl 2-methyl-5-oxo-4-phenyl-1,4,5,6,7,8-hexahydroquinoline-3-carboxylate (5a). Mp 241$242{ }^{\circ} \mathrm{C} ;{ }^{1} \mathrm{H}$ NMR $\left(\mathrm{CDCl}_{3}, 400 \mathrm{MHz}\right): \delta=7.30-7.26(\mathrm{~m}, 2 \mathrm{H}), 7.22-7.19(\mathrm{~m}, 2 \mathrm{H}), 7.13-7.10(\mathrm{~m}$, $1 \mathrm{H}), 6.06(\mathrm{~s}, 1 \mathrm{H}), 5.08(\mathrm{~s}, 1 \mathrm{H}), 4.05$ (q, $J=6.8 \mathrm{~Hz}, 2 \mathrm{H}), 2.45-2.31(\mathrm{~m}, 7 \mathrm{H}), 2.09-1.85(\mathrm{~m}, 2 \mathrm{H})$, 1.19 (t, $J=6.8 \mathrm{~Hz}, 3 \mathrm{H}) ;{ }^{13} \mathrm{C} \mathrm{NMR}\left(\mathrm{CDCl}_{3}, 100 \mathrm{MHz}\right): \delta=195.6,167.4,149.5,147.1,143.2$, $128.0,127.9,126.0,113.4,106.1,59.7,37.1,36.4,27.5,21.1,19.2,14.2$; IR (KBr): $v=3283$, 1703, 1695, $1614 \mathrm{~cm}^{-1}$; EIMS: $m / z(\%)=311\left(\mathrm{M}^{+}\right)$; Anal. Calcd for $\mathrm{C}_{19} \mathrm{H}_{21} \mathrm{NO}_{3}$ : C, 73.29; $\mathrm{H}$, $6.80 ; \mathrm{N}, 4.50$. Found: C, 73.35; H, 6.88; N, 4.56.

Ethyl 2-methyl-5-oxo-4-(2-nitrophenyl)-1,4,5,6,7,8-hexahydroquinoline-3-carboxylate (5b). Mp 191-192 ${ }^{\circ} \mathrm{C} ;{ }^{1} \mathrm{H}$ NMR $\left(\mathrm{CDCl}_{3}, 400 \mathrm{MHz}\right): \delta=7.71-7.68(\mathrm{~m}, 1 \mathrm{H}), 7.61-7.40(\mathrm{~m}, 2 \mathrm{H}), 7.40(\mathrm{~s}$, $1 \mathrm{H}), 7.25-7.22(\mathrm{~m}, 1 \mathrm{H}), 5.88(\mathrm{~s}, 1 \mathrm{H}), 4.08-4.02(\mathrm{~m}, 2 \mathrm{H}), 2.51-2.21(\mathrm{~m}, 7 \mathrm{H}), 2.00-1.75(\mathrm{~m}, 2 \mathrm{H})$, $1.12(\mathrm{t}, J=7.1 \mathrm{~Hz}, 3 \mathrm{H}) ;{ }^{13} \mathrm{C} \mathrm{NMR}\left(\mathrm{CDCl}_{3}, 100 \mathrm{MHz}\right): \delta=196.0,167.2,152.2,148.2,145.3$, 141.8, 132.6, 131.0, 126.4, 123.5, 111.9, 104.6, 59.7, 36.6, 32.4, 26.85, 20.8, 18.7, 13.9; IR $(\mathrm{KBr}): v=3285,1705,1692,1620,1525 \mathrm{~cm}^{-1}$; EIMS: $m / z(\%)=356\left(\mathrm{M}^{+}\right)$; Anal. Calcd for $\mathrm{C}_{19} \mathrm{H}_{20} \mathrm{~N}_{2} \mathrm{O}_{5}$ : C, 64.04; H, 5.66; N, 7.86. Found: C, 64.11; H, 5.71; N, 7.90.

Ethyl 2-methyl-5-oxo-4-(4-nitrophenyl)-1,4,5,6,7,8-hexahydroquinoline-3-carboxylate (5c). $\mathrm{Mp} 205-206{ }^{\circ} \mathrm{C} ;{ }^{1} \mathrm{H}$ NMR $\left(\mathrm{CDCl}_{3}, 400 \mathrm{MHz}\right): \delta=7.30-7.26(\mathrm{~m}, 2 \mathrm{H}), 7.22-7.19(\mathrm{~m}, 2 \mathrm{H}), 6.06(\mathrm{~s}$, $1 \mathrm{H}), 5.08(\mathrm{~s}, 1 \mathrm{H}), 4.05(\mathrm{q}, J=6.8 \mathrm{~Hz}, 2 \mathrm{H}), 2.45-2.31(\mathrm{~m}, 7 \mathrm{H}), 2.09-1.85(\mathrm{~m}, 2 \mathrm{H}), 1.19(\mathrm{t}, J=6.8$ $\mathrm{Hz}, 3 \mathrm{H}) ;{ }^{13} \mathrm{C} \mathrm{NMR}\left(\mathrm{CDCl}_{3}, 100 \mathrm{MHz}\right): \delta=196.2,167.1,154.9,151.6,146.4,144.8,134.9$, 129.0, 111.9, 104.5, 60.1, 37.1, 36.8, 27.2, 20.9, 19.2, 14.1; IR (KBr): $v=3287,1708,1696$, 1623, $1518 \mathrm{~cm}^{-1}$; EIMS: $m / z(\%)=356\left(\mathrm{M}^{+}\right)$; Anal. Calcd for $\mathrm{C}_{19} \mathrm{H}_{20} \mathrm{~N}_{2} \mathrm{O}_{5}: \mathrm{C}, 64.04 ; \mathrm{H}, 5.66 ; \mathrm{N}$, 7.86. Found: C, 64.10; H, 5.70; N, 7.89.

Ethyl 2-methyl-5-oxo-4-(4-methylphenyl)-1,4,5,6,7,8-hexahydroquinoline-3-carboxylate (5d). Mp 241-242 ${ }^{\circ} \mathrm{C} ;{ }^{1} \mathrm{H} \mathrm{NMR}\left(\mathrm{CDCl}_{3}, 400 \mathrm{MHz}\right): \delta=7.32(\mathrm{~s}, 1 \mathrm{H}), 7.19(\mathrm{~d}, J=8.0 \mathrm{~Hz}, 2 \mathrm{H})$, $7.01(\mathrm{~d}, J=8.0 \mathrm{~Hz}, 2 \mathrm{H}), 5.05(\mathrm{~s}, 1 \mathrm{H}), 4.07$ (q, $J=7.2 \mathrm{~Hz}, 2 \mathrm{H}), 2.50-2.21(\mathrm{~m}, 10 \mathrm{H}), 2.10-1.80$ $(\mathrm{m}, 2 \mathrm{H}), 1.21(\mathrm{t}, J=7.2 \mathrm{~Hz}, 3 \mathrm{H}) ;{ }^{13} \mathrm{C} \mathrm{NMR}\left(\mathrm{CDCl}_{3}, 100 \mathrm{MHz}\right): \delta=196.3,167.6,151.4,144.5$, $143.9,135.4,129.6,128.5,127.7,112.7,105.8,59.7,37.0,35.8,26.9,20.9,18.9,14.1$; IR (KBr): 
$v=3272,1700,1690,1645 \mathrm{~cm}^{-1}$; EIMS: $m / z(\%)=325\left(\mathrm{M}^{+}\right)$; Anal. Calcd for $\mathrm{C}_{20} \mathrm{H}_{23} \mathrm{NO}_{3}$ : C, 73.82; H, 7.12; N, 4.30. Found: C, 73.86; H, 7.16; N, 4.33.

Ethyl 2-methyl-5-oxo-4-(4-methoxyphenyl)-1,4,5,6,7,8-hexahydroquinoline-3-carboxylate (5e). Mp 194-195 ${ }^{\circ} \mathrm{C} ;{ }^{1} \mathrm{H} \mathrm{NMR}\left(\mathrm{CDCl}_{3}, 400 \mathrm{MHz}\right): \delta=7.21(\mathrm{~d}, J=8.5 \mathrm{~Hz}, 2 \mathrm{H}), 7.10(\mathrm{~d}, J=8.5$ $\mathrm{Hz}, 2 \mathrm{H}), 5.92(\mathrm{~s}, 1 \mathrm{H}), 5.04(\mathrm{~s}, 1 \mathrm{H}), 4.06(\mathrm{q}, J=6.8 \mathrm{~Hz}, 2 \mathrm{H}), 3.74(\mathrm{~s}, 3 \mathrm{H}), 2.58-2.31(\mathrm{~m}, 7 \mathrm{H})$, 2.11-1.82 (m, 2H), $1.20(\mathrm{t}, J=6.8 \mathrm{~Hz}, 3 \mathrm{H}) ;{ }^{13} \mathrm{C} \mathrm{NMR}\left(\mathrm{CDCl}_{3}, 100 \mathrm{MHz}\right): \delta=195.7,167.5$, $157.7,149.1,142.9,139.8,128.9,113.7,113.3,106.4,59.8,55.1,37.0,35.4,27.5,21.1,19.4$, 14.2; IR $(\mathrm{KBr}): v=3268,1700,1688,1616 \mathrm{~cm}^{-1}$; EIMS: $m / z(\%)=341\left(\mathrm{M}^{+}\right)$; Anal. Calcd for $\mathrm{C}_{20} \mathrm{H}_{23} \mathrm{NO}_{4}$ : C, 70.36; H, 6.79; N, 4.10. Found: C, 70.42; H, 6.83; N, 4.12.

Ethyl 2-methyl-5-oxo-4-(4-chlorophenyl)-1,4,5,6,7,8-hexahydroquinoline-3-carboxylate (5f). Mp 234-235 ${ }^{\circ} \mathrm{C} ;{ }^{1} \mathrm{H} \mathrm{NMR}\left(\mathrm{CDCl}_{3}, 400 \mathrm{MHz}\right): \delta=7.25(\mathrm{~d}, J=8.8 \mathrm{~Hz}, 2 \mathrm{H}), 7.18(\mathrm{~d}, J=8.8 \mathrm{~Hz}$, $2 \mathrm{H}), 6.30(\mathrm{~s}, 1 \mathrm{H}), 6.05(\mathrm{~s}, 1 \mathrm{H}), 4.05$ (q, $J=7.2 \mathrm{~Hz}, 2 \mathrm{H}), 2.48-2.31(\mathrm{~m}, 7 \mathrm{H}), 2.11-1.83(\mathrm{~m}, 2 \mathrm{H})$, $1.18(\mathrm{t}, J=7.2 \mathrm{~Hz}, 3 \mathrm{H}) ;{ }^{13} \mathrm{C} \mathrm{NMR}\left(\mathrm{CDCl}_{3}, 100 \mathrm{MHz}\right): \delta=195.8,167.2,150.3,145.6,143.5$, 131.6, 129.4, 128.0, 113.0, 105.8, 59.9, 36.8, 36.1, 27.4, 21.0, 19.3, 14.2; IR (KBr): $v=3270$, 1700, 1685, $1622 \mathrm{~cm}^{-1}$; EIMS: $m / z(\%)=345\left(\mathrm{M}^{+}\right)$; Anal. Calcd for $\mathrm{C}_{19} \mathrm{H}_{20} \mathrm{ClNO}_{3}$ : C, 65.99; $\mathrm{H}$, 5.83 ; N, 4.05. Found: C, 66.04; H, 5.87; N, 4.08 .

Ethyl 2-methyl-5-oxo-4-(4-fluorophenyl)-1,4,5,6,7,8-hexahydroquinoline-3-carboxylate (5g). Mp 243-244 ${ }^{\circ} \mathrm{C} ;{ }^{1} \mathrm{H}$ NMR (DMSO- $\left.d_{6}, 400 \mathrm{MHz}\right): \delta=7.27-7.23(\mathrm{~m}, 2 \mathrm{H}), 6.89-6.85(\mathrm{~m}, 2 \mathrm{H}), 6.03$ $(\mathrm{s}, 1 \mathrm{H}), 5.07(\mathrm{~s}, 1 \mathrm{H}), 4.05(\mathrm{q}, J=7.2 \mathrm{~Hz}, 2 \mathrm{H}), 2.70-2.21(\mathrm{~m}, 7 \mathrm{H}), 2.11-1.82(\mathrm{~m}, 2 \mathrm{H}), 1.17(\mathrm{t}, J=$ $7.2 \mathrm{~Hz}, 3 \mathrm{H}) ;{ }^{13} \mathrm{C}$ NMR (DMSO- $\left.d_{6}, 100 \mathrm{MHz}\right): \delta=195.1,167.2,151.9,145.6,144.5,129.6$, $129.4,114.9$, 111.5, 103.8, 59.5, 37.1, 35.6, 26.8, 21.3, 18.8, 14.6; IR (KBr): $v=3268,1702$, 1688, $1620 \mathrm{~cm}^{-1}$; EIMS: $m / z(\%)=329\left(\mathrm{M}^{+}\right)$; Anal. Calcd for $\mathrm{C}_{19} \mathrm{H}_{20} \mathrm{FNO}_{3}: \mathrm{C}, 69.29 ; \mathrm{H}, 6.12 ; \mathrm{N}$, 4.25. Found: C, 69.34; H, 6.17; N, 4.28.

Ethyl 2-methyl-5-oxo-4-(4-hydrophenyl)-1,4,5,6,7,8-hexahydroquinoline-3-carboxylate (5h). Mp 221-222 ${ }^{\circ} \mathrm{C} ;{ }^{1} \mathrm{H}$ NMR (Acetone- $\left.d_{6}, 400 \mathrm{MHz}\right): \delta=7.07(\mathrm{~d}, J=8.4 \mathrm{~Hz}, 2 \mathrm{H}), 6.64(\mathrm{~d}, J=8.4$ $\mathrm{Hz}, 2 \mathrm{H}), 5.05(\mathrm{~s}, 1 \mathrm{H}), 4.02(\mathrm{q}, J=7.2 \mathrm{~Hz}, 2 \mathrm{H}), 2.53-2.00(\mathrm{~m}, 2 \mathrm{H}), 2.34(\mathrm{~s}, 3 \mathrm{H}), 2.23-2.19(\mathrm{~m}$, $2 \mathrm{H}), 2.00-1.72(\mathrm{~m}, 2 \mathrm{H}), 1.18(\mathrm{t}, J=7.2 \mathrm{~Hz}, 3 \mathrm{H}) ;{ }^{13} \mathrm{C}$ NMR (Acetone- $\left.d_{6}, 100 \mathrm{MHz}\right): \delta=195.7$, $168.4,156.5,151.5,145.3,140.8,130.2,115.7,113.8,106.3,60.2,38.1,36.7,27.7,22.4$, 19.2, 15.0; IR $(\mathrm{KBr}): v=3300,1700,1688,1617 \mathrm{~cm}^{-1}$; EIMS: $m / z(\%)=327\left(\mathrm{M}^{+}\right)$; Anal. Calcd for $\mathrm{C}_{19} \mathrm{H}_{21} \mathrm{NO}_{4}$ : C, 69.71; H, 6.47; N, 4.28. Found: C, 69.75; H, 6.49; N, 4.34.

Ethyl 2-methyl-5-oxo-4-(2-furyl)-1,4,5,6,7,8-hexahydroquinoline-3-carboxylate (5i). Mp 211-212 ${ }^{\circ} \mathrm{C} ;{ }^{1} \mathrm{H} \mathrm{NMR}\left(\mathrm{CDCl}_{3}, 400 \mathrm{MHz}\right): \delta=7.30-7.26(\mathrm{~m}, 1 \mathrm{H}), 7.22-7.19(\mathrm{~m}, 1 \mathrm{H}), 7.13-7.10$ $(\mathrm{m}, 1 \mathrm{H}), 6.06(\mathrm{~s}, 1 \mathrm{H}), 5.08(\mathrm{~s}, 1 \mathrm{H}), 4.05(\mathrm{q}, J=6.8 \mathrm{~Hz}, 2 \mathrm{H}), 2.45-2.31(\mathrm{~m}, 7 \mathrm{H}), 2.09-1.85(\mathrm{~m}$, $2 \mathrm{H}), 1.19(\mathrm{t}, J=6.8 \mathrm{~Hz}, 3 \mathrm{H}) ;{ }^{13} \mathrm{C} \mathrm{NMR}\left(\mathrm{CDCl}_{3}, 100 \mathrm{MHz}\right): \delta=195.6,167.3,157.9,150.6,144.2$, 140.8, 110.1, 106.1, 104.8, 103.0, 59.9, 37.0, 30.4, 27.5, 21.0, 19.4, 14.3; IR (KBr): v = 3278, 1704, 1690, $1614 \mathrm{~cm}^{-1}$; EIMS: $\mathrm{m} / z(\%)=301\left(\mathrm{M}^{+}\right)$; Anal. Calcd for $\mathrm{C}_{17} \mathrm{H}_{19} \mathrm{NO}_{4}$ : C, 67.76; $\mathrm{H}$, 6.36; N, 4.65. Found: C, 67.81; H, 6.41; N, 4.68. 


\section{Acknowledgements}

We gratefully acknowledge financial support from the National Natural Science Foundation of China (No. 20562005) and NSF of Jiangxi Province (No. 0620021).

\section{References}

1. Mauzeral, D.; Westheimer, F. H. J. Am. Chem. Soc. 1955, 77, 2261.

2. (a) Kawase, M.; Shah, A.; Gaveriya, H.; Motohashi, N.; Sakagami, H.; Varga A.; Molnar, J. Bioorg. Med. Chem. 2002, 10, 1051. (b) Suarez, M.; Verdecia, Y.; Illescas, B.; MartinezAlvarez, R.; Avarez, A.; Ochoa, E.; Seoane, C.; Kayali, N.; Martin, N. Tetrahedron 2003, 59, 9179. (c) Sabitha, G.; Reddy, G. S. K. K.; Reddy, C. S.; Yadav, J. S. Tetrahedron Lett. 2003, 44, 4129. (d) Sawada, Y.; Kayakiri, H.; Abe, Y.; Mizutani, T.; Inamura, N.; Asano, M.; Hatori, C.; Aramori, I.; Oku, T.; Tanaka, H. J. Med. Chem. 2004, 47, 2853. (e) Shan, R.; Velazquez, C.; Knaus, E. E. J. Med. Chem. 2004, 47, 254.

3. Zhang, D.; Wu, L.-Z.; Zhou, L.; Han, X.; Yang, Q.-Z.; Zhang, L.-P.; Tung, C.-H. J. Am. Chem. Soc. 2004, 126, 3440.

4. Love, B.; Snader, K. M. J. Org. Chem. 1965, 30, 1914.

5. (a) Khadikar, B. M.; Gaikar, V. G.; Chitnavis, A. A. Tetrahedron Lett. 1995, 36, 8083. (b) Ohberg, L.; Westman, J. Synlett 2001, 1296. (c) Tu, S.-J.; Zhou, J.-F.; Deng, X.; Cai, P.-J.; Wang, H. Feng, J.-C. Chin. J. Org. Chem. 2001, 21, 313. (d) Agarwal, A.; Chauhan, P. M. S. Tetrahedron Lett. 2005, 46, 1345.

6. (a) Ji, S.-J.; Jiang, Z.-Q.; Lu, J.; Loh, T.-P. Synlett 2004, 831. (b) Sridhar, R.; Perumal, P. T. Tetrahedron 2005, 61, 2465.

7. (a) Phillips, A. P. J. Am. Chem. Soc. 1949, 71, 4003. (b) Anderson, G. Jr. Berkelhammer, G. J. Am. Chem. Soc. 1958, 80, 992. (c) Singh, H.; Chimni, D. S. S.; Kumar, S. Tetrahedron 1995, 51, 12775. (d) Gordeev, M. F.; Patel, D. V.; Gordon, E. M. J. Org. Chem. 1996, 61, 924. (e) Breitenbucher, J. G.; Figliozzi, G. Tetrahedron Lett. 2000, 41, 4311. (f) Liang, J.C.; Yeh, J.-L.; Wang, C.-S.; Liou, S.-F.; Tasi, C.-H.; Chen, I.-J. Bioorg. Med. Chem. 2002, 10, 719. (g) Dondoni, A.; Massi, A.; Minghini, E.; Sabbatini, S.; Bertoasi, V. J. Org. Chem. 2003, 68, 6172. (h) Dondoni, A.; Massi, A.; Minghini, E.; Bertoasi, V. Tetrahedron 2004, 60, 2311. (i) Tewari, N.; Dwivedi, N.; Tripathi, R. P. Tetrahedron Lett. 2004, 45, 9011. (j) Zolfigol, M. A.; Safaiee, M. Synlett 2004, 827. (k) Moseley, J. D. Tetrahedron Lett. 2005, $46,3179$.

8. Sabitha, G.; Reddy, G. S. K. K.; Reddy, Ch. S.; Yadav, J. S. Tetrahedron Lett. 2003, 44, 4129.

9. Wang, L.-M.; Sheng, J.; Zhang, L.; Han, J.-W.; Fan, Z.-Y.; Tian, H.; Qian, C.-T. Tetrahedron 2005, 61, 1539.

10. Ko, S.; Yao, C.-F. Tetrahedron 2006, 62, 7293. 
11. Ko, S.; Sastry, M. N. V.; Lin, C.; Yao, C.-F. Tetrahedron Lett. 2005, 46, 5771.

12. (a) Gordeev, M. F.; Patel, D. V.; Gordon, E. M. J. Org. Chem. 1996, 61, 924. (b) Breitenbucher, J. G.; Figliozzi, G. Tetrahedron Lett. 2000, 41, 4311. (c) Rodríguez, H.; Reyes, O.; Suárez, M.; Garay, H. E.; Pérez, R.; Cruz, L. J.; Verdecia, Y.; Martín, N.; Seoane, C. Tetrahedron Lett. 2002, 43, 439.

13. (a) Gravert, D. J.; Janda, K. D. Chem. Rev. 1997, 97, 489. (b) Wentworth, P.; Janda, K. D. Chem. Commun. 1999, 1917. (c) Toy, P. H.; Tanda, K. D. Acc. Chem. Res. 2000, 33, 546.

14. Sheng, S.-R.; Huang, P.-G.; Wang, Q.; Huang, R.; Liu, X.-L. Synth. Commun. 2006, 36, 3175 .

15. Data for compound 6a: $\mathrm{Mp} 294-296{ }^{\circ} \mathrm{C} ;{ }^{1} \mathrm{H}$ NMR $\left(\mathrm{CDCl}_{3}, 400 \mathrm{MHz}\right): \delta=7.31-7.20(\mathrm{~m}$, $4 \mathrm{H}), 7.14-7.12(\mathrm{~m}, 1 \mathrm{H}), 5.08(\mathrm{~s}, 1 \mathrm{H}), 2.68-2.57(\mathrm{~m}, 4 \mathrm{H}), 2.37-2.31(\mathrm{~m}, 3 \mathrm{H}), 2.06-1.96(\mathrm{~m}$, $2 \mathrm{H}) ;{ }^{13} \mathrm{C} \mathrm{NMR}\left(\mathrm{CDCl}_{3}, 100 \mathrm{MHz}\right): \delta=196.7,164.0,150.3,147.4,144.9,128.3,128.1$, 126.4, 116.9, 107.8, 37.0, 31.6, 29.7, 21.4, 20.3; IR (KBr): 3146, 1709, 1674, $1620 \mathrm{~cm}^{-1}$. EIMS: $m / z(\%)=283\left(\mathrm{M}^{+}\right)$; Anal. Calcd for $\mathrm{C}_{17} \mathrm{H}_{17} \mathrm{NO}_{3}$ : C, 72.07; H, 6.05; N, 4.94. Found: C, 72.12; H, 6.11; N, 4.99 . 